\title{
Weighted Integral Rotation and Translation for Touch Interaction
}

\author{
Gun A. Lee* and Mark Billinghurst ${ }^{\dagger}$ \\ The Human Interface Technology Laboratory New Zealand, University of Canterbury
}

\section{Introduction}

Touch based interaction is popular in graphical user interface (GUI) systems, as it provides natural and intuitive direct manipulation. Rotation and translation are basic tasks for manipulating graphical objects and various touch based interaction techniques has been investigated for doing this [Hancock et al. 2006]. In early GUI systems, users had to perform rotation and translation independently by switching between the two manipulation modes either using a menu system or by manipulating different widgets that in many cases make the interface visually cluttered. Recently, two-finger gestures have become common in multi-touch interfaces to perform rotation, translation, and even scaling, simultaneously, without visual clutter. However, there can be ergonomic problems when the user has to rotate objects in large angle [Hoggan et al. 2013], which causes strain on user's wrist. As a result users tend to split and perform the manipulation in multiple steps, which might not be suitable for certain applications, such as puppeteering based animation tools.

\section{Weighted Integral Rotation and Translation}

With the Integral Rotation and Translation (RNT) [BeaudouinLafon 2001; Kruger et al. 2005] technique, users can perform both rotation and translation in an integrated way using only one finger. Hence it does not have the ergonomic problems found in two-finger gestures. RNT mimics physical manipulation of flat objects (e.g. paper) on a table, where the friction between the object and the table surface generates torque around the object's center of gravity. When an object is dragged from $T_{0}$ to $T_{1}$ using the RNT technique, it is also rotated about $T_{1}$ by the angle $\theta$ ( $\left.=\angle T_{0} O_{0} T_{1}=\angle O_{1} T_{1} O_{0}\right)$, so that the final center of gravity $O_{2}$ is on the line of $O_{0} T_{1}$ (see left side of Figure 1).
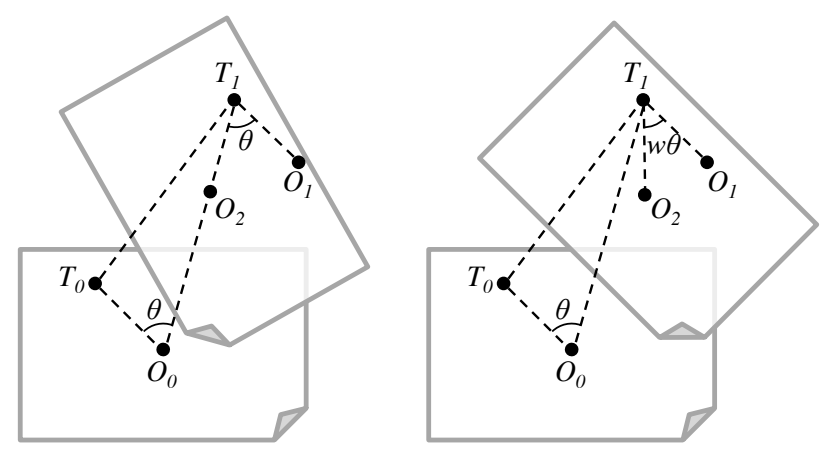

Figure 1: $R N T$ (left) and $w R N T$ (right).

While the RNT method provides intuitive integration of rotation and translation, rotation cannot be applied when $T_{0}=O_{0}$. To avoid this, Kruger et al. [2005] had a region around the center of gravity where only translation is applied. However, this could be confusing to the user as the context of manipulation switches suddenly at the

*e-mail:gun.lee@hitlabnz.org

†e-mail:mark.billinghurst@hitlabnz.org

Permission to make digital or hard copies of part or all of this work for personal or classroom use is granted without fee provided that copies are not made or distributed for commercial advantage and tha copies bear this notice and the full citation on the first page. Copyrights for third-party components of this work must be honored. For all other uses, contact the Owner/Autho

SIGGRAPH Asia 2013, November $19-22$,

ACM $978-1-4503-2511-0 / 13 / 11$ boundary of such region. One solution is to visually mark the region to help users to recognize the boundary, but this also increases onscreen visual clutter.

In our research, we overcome these problems by introducing a weight factor to the amount of rotation integrated to translation. We call the method Weighted Integral Rotation and Translation (wRNT). As shown on the right side of Figure 1, we multiply the weight $w(0 \leq w \leq 1)$ to the rotation angle $\theta$ to control the amount of rotation. The weight is proportional to the distance $d$ between the touch point $T_{0}$ and the center of gravity $O_{0}$, and it can be calculated with a simple function such as the proportion of $d$ to the radius of the object $r$. To create a smoother transition, the weight can be mapped using sigmoid functions such as a partial sine wave or logistic function. The weight function can also be used when $T_{0} T_{1}$ is perpendicular to $O_{0} T_{0}$. In this case we set the value to 1 to support full rotation.

Figure 2 shows our implementation of the wRNT technique on an Android tablet. Dragging different points on the same object results in different weights being given to the integrated rotation while translating the object. In the future, we plan to conduct a user study to investigate the strengths and weaknesses of the technique, and apply it to a puppeteering based animation tool.

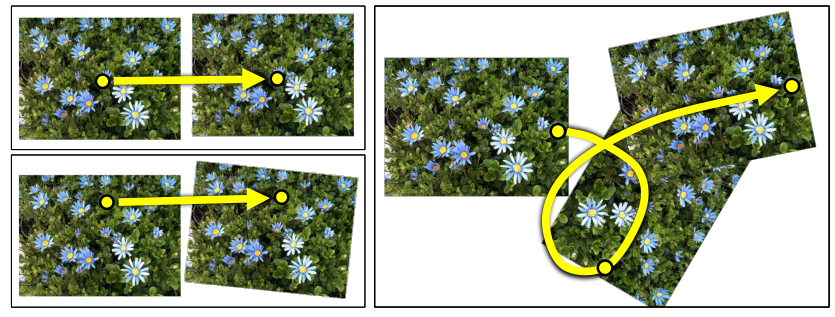

Figure 2: Dragging different points on the same object using $w R N T$ (yellow dots and arrows show touch points and dragged directions).

\section{References}

Beaudouin-LAFon, M. 2001. Novel interaction techniques for overlapping windows. In Proceedings of the 14th annual ACM symposium on User interface software and technology, UIST '01, 153-154.

Hancock, M., Vernier, F., Wigdor, D., Carpendale, S., AND SHEN, C. 2006. Rotation and translation mechanisms for tabletop interaction. In Horizontal Interactive Human-Computer Systems, 2006. TableTop 2006. First IEEE International Workshop on, 79-86.

Hoggan, E., Williamson, J., Oulasvirta, A., Nacenta, M., Kristensson, P. O., AND Lehtiö, A. 2013. Multi-touch rotation gestures: performance and ergonomics. In Proceedings of the SIGCHI Conference on Human Factors in Computing Systems, CHI '13, 3047-3050.

Kruger, R., Carpendale, S., Scott, S. D., and Tang, A. 2005. Fluid integration of rotation and translation. In Proceedings of the SIGCHI Conference on Human Factors in Computing Systems, CHI '05, 601-610. 